\title{
Preoperative diagnosis of strangulated obturator hernia
}

\author{
Emmunuel QuAYe Archampong* \\ B.Sc., M.B., F.R.C.S., F.R.C.S.E. \\ Surgical Registrar, \\ Queen Elizabeth II Hospital, Welwyn Garden City, Herts.
}

\section{Summary}

Six cases of strangulated obturator hernia seen over a period of 8 months in a sixty-bed surgical unit are reported. The sex ratio was five females to one male. There was no mortality.

The importance of preoperative diagnosis or suspicion of obturator hernia is stressed. In this radiology may be of help.

Treatment is briefly discussed.

\section{Introduction}

Reports are unanimous in commenting on the rarity of obturator herniation and the diagnosis is indeed seldom made before the onset of acute symptoms (Desmond \& Hutter, 1948 ; Harper \& Holt, 1956). The writings of Wakeley (1939) and Watson (1948) have stimulated surgical interest in the preoperative diagnosis of strangulated obturator hernia but the recent assertion (Bailey, 1958) that there are only 500 cases on record would indicate that the condition has still to attain its true perspective in surgical practice. Over a period of 8 months on a sixty-bed unit in a young expanding population, six cases were treated. This raises the suspicion that this condition is commoner than usually conceded and that missed cases may exist in that group of intestinal obstruction which settle with conservative management. Although the diagnosis was considered in three of the six cases, it was established preoperatively only on one occasion.

\section{Case reports}

Case 1

An 87-year-old man complained of colicky peri-umbilical pain and vomiting of increasing frequency; at the time of admission he was vomiting half-hourly. He had been constipated for 4 days. Five months earlier he had had a

\footnotetext{
* Present address: School of Medicine, Accra, Ghana.
}

less severe episode which had settled spontaneously.

Examination showed a moderately dehydrated old man with a tympanitic abdomen devoid, however, of abnormal masses. The hernial orifices were free of ruptures. The bowel sounds were violent suggesting intestinal obstruction which was confirmed radiologically by numerous fluid levels in the lower ileum. Conservative measures were unavailing and with a presumptive diagnosis of internal herniation laparotomy was performed. The approach was through a right paramedian incision revealing the cause of obstruction as a strangulated left obturator hernia of the Richter type. It was reduced without difficulty, the bowel being viable. The sac was excised and its neck closed. His post-operative course was uneventful.

\section{Case 2}

An 80-year-old woman was admitted with generalized colicky abdominal pain and vomiting of 2 days' duration. She had experienced pain intermittently down the left thigh and in the left knee and had been constipated for 2 days. In 1946 she had an uncomplicated appendicectomy.

On examination she was a white-haired lady showing no evidence of recent weight loss; she was slightly dehydrated. Her abdomen was distended and generally tender, but no masses could be felt. Bowel sounds were obstructive. Vaginal and rectal examinations were normal.

The obstructive presentation was confirmed radiologically by fluid levels in the erect films. Adhesions from her appendicectomy were thought responsible but as she showed no improvement on gastro-intestinal suction and fluid therapy she was subjected to laparotomy, the possibility of an obturator hernia having been raised. Through a right paramedian incision it was noticed that the small bowel was obstructed by a strangulated left obturator hernia which 
was reduced with some difficulty after stretching the neck of the obturator canal. The knuckle of bowel required resection with end-to-end anastomosis. Urinary infection occurring post-operatively responded well to ampicillin.

\section{Case 3}

An 84-year-old woman who had suffered with vague abdominal pains and obstinate constipation for many years, was admitted on 7 January 1967 with sudden onset of colicky lower abdominal pain frequently accompanied by pain down both legs, particularly the left. In 1956 and 1964 she had had similar attacks which had necessitated gastro-enterological investigations elsewhere including barium meal and cholecystogram with negative results.

Examination revealed an anaemic ill-looking but well-covered and hydrated old lady. Her abdomen was distended and bowel sounds were hyperactive. External herniae were absent and vaginal and rectal examinations were negative.

She was thought to have incomplete intestinal obstruction, a view confirmed by the erect X-ray

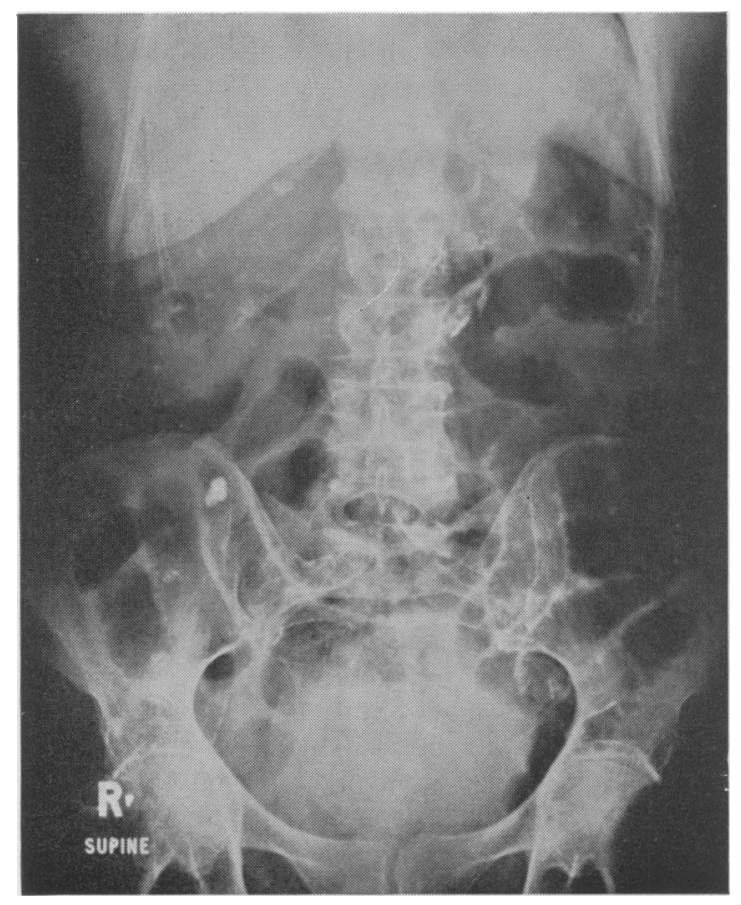

FIG. 1. Plain X-ray of the abdomen showing small bowel shadows in the left iliac fossa close to the superior ramus of the pubic bone. films which also showed small bowel shadows well down in the left iliac fossa close to the superior ramus (Fig. 1) of the pubic bone, a most unusual radiological finding. This raised a suspicion of an obstructed obturator hernia although adhesions from a previous appendicectomy were still considered the more probable cause.

Management with gastro-intestinal suction produced immediate improvement which was shortlived with recrudescence of symptoms in $36 \mathrm{hr}$. Laparotomy was performed through a left paramedian incision revealing a strangulated left obturator hernia. Reduction presented no difficulty and the sac which had become inverted in the process was transfixed. Post-operative course was uneventful.

\section{Case 4}

A 76-year-old housewife who had had intermittent abdominal pains for 5 years was admitted in a violent exacerbation with vomiting; her bowels had been confined for 4 days.

On examination she was dehydrated and obviously ill. Estimation of serum electrolytes showed marked deficiencies in sodium, chloride and potassium which were corrected by appropriate therapy. Her abdomen was particularly distended in the hypogastrium with loud bowel sounds. Partly on suspicion of internal hernia and because the obstruction was judged advanced laparotomy was performed within an hour of admission revealing a strangulated right obturator hernia. The affected bowel was readily reduced and her post-operative course was marred only by superficial thrombophlebitis.

\section{Case 5}

A 64-year-old woman was admitted to hospital with acute abdominal pain of $36 \mathrm{hr}$ duration; she had been vomiting for $12 \mathrm{hr}$ and had been constipated for 2 days.

On examination she was an ill but well-covered lady with the classic silky-white hair. Her abdomen was rigid and bowel sounds were obstructive but there were no external herniae.

She was operated on forthwith whereupon a knuckle of ileum herniated through the left obturator foramen was found. Owing to the poor state of the patient it was decided not to excise the sac. She made a satisfactory recovery and was discharged on the 10th day.

She was readmitted 3 months later in another attack of intestinal obstruction. While adhesions could not be excluded recurrent obturator hernia was thought highly probable. Laparotomy was therefore not delayed. The hernia had indeed 
recurred and under better circumstances the sac was on this occasion excised.

\section{Case 6}

A 75-year-old woman was admitted with sudden onset of colicky lower abdominal pain and vomiting. For many years she had had a right femoral hernia for which she had declined operation. In answer to questions she admitted pain down the right leg but insisted that it was rheumatic.

On examination she was clearly obstructed. The femoral hernia was tense and tender and was considered a sufficient cause for the clinical picture. At operation it was found through the low pararectal approach of McEvedy that there were in fact two strangulated herniae; the femoral sac contained devitalized omentum, while just below it was a knuckle of bowel trapped in a right obturator hernial sac, Richter fashion. Both herniae were repaired without difficulty; recovery was uninterrupted.

\section{Discussion}

Preoperative diagnosis of strangulated obturator hernia, or at least serious consideration of the condition, is essential to improvement in results of treatment. In the very rare event of a palpable lump in the groin without symptoms, the difficulty would be in the differentiation from femoral hernia. No such case was seen but Desmond \& Hutter's (1948) suggestion of eliciting tenderness by pressure on the pubic ramus above the lump would sound attractive.

More puzzling are the cases with prolonged subacute or chronic presentation taking the form of incomplete or repeated obstructive episodes (as in Case 3), where the patients complain of recurrent abdominal pain sometimes with pain in the distribution of the obturator nerve and constipation. Patient No. 3 had, until the acute obstruction, accepted her discomfort as part of the routine of life.

The patient's advanced age, body habit, distribution of the pain in the course of the anterior branch of the obturator nerve (Howship-Romberg sign) with or without tenderness in Scarpa's triangle and the pelvic findings should direct attention to the obturator canal.

It is in strangulation that a preoperative consideration of obturator herniation is most urgent and has a claim to priority whenever the above symptom-complex is accompanied by acute intestinal obstruction. Discrepancies may arise but should not exclude the diagnosis if the overall picture indicated it. Thus among the cases reported, two patients were rather obese (Cases
3 and 5) with no recent weight loss; this did not affect the clinical interpretation of the cases. The Howship-Romberg sign may prove quite difficult to differentiate from the much commoner rheumatic pains in the legs of the population at risk. Its significance becomes questionable when bilateral and not strictly medial in position. In the two cases where it contributed to diagnosis the pain followed precisely the anterior branch of the obturator nerve.

Plain X-rays of the abdomen may be of help, as in Case 3, where the small-bowel contents of the sac were demonstrated in close proximity to the superior ramus of the pubic bone. Indeed on the left side the sign does seriously strengthen the suspicion of obturator hernia as small bowel shadows seldom occupy the lower reaches of the left iliac fossa. On the right side this sign may still have an important role.

All but one of the cases (Case 6) were subjected to laparotomy, and where a preoperative diagnosis had been made (case 3 ) better planning of the operation in the siting of the incision and speed of execution were rewarded by freedom from post-operative morbidity and early recovery. Striking was the ease with which the reduction was effected in most of the hernias stretching of the neck was necessary in one case only, and this was the only one that requiredo bowel resection. This had been the experience of Desmond \& Hutter (1948), although Kwong \& Ong (1966) had to resect four of their six cases and a fifth was perforated requiring simple closure.

The importance of removal of the sac or some form of repair of the defect was borne out by the fact that in the one case that the sac was left behind because the poor general condition of the patient demanded speedy completion of the operation, the hernia recurred and restrangulated. In this I am in agreement with Desmond \& Hutter (1948) that removal of the sac is all that needs to be done. In many cases the sac is rather shallow and inverts when its fundus is seized with a haemostat and gentle traction applied; the neck can then be transfixed. Due regard should be given to surrounding structures - the obturator artery and nerve and bladder.

Case 6 is worthy of note as a double hernia ; a large femoral and a small obturator both strangulated. The approach had been by the low pararectal incision of McEvedy and the ease with which the second hernia was dealt with stresses the advantage of this approach for strangulated femoral herniae. This also demonstrates the importance of thorough examination of the bowel in cases of intestinal obstruction. 


\section{Acknowledgments}

I would like to thank Mr. Gordon F. Cassie, F.R.C.S., CH.M., and Mr W. Donald Bedford, F.R.C.s., Senior Consulting Surgeons to the Queen Elizabeth II Hospital, for permission to study cases admitted under their care and also for making valuable comments on the manuscript.

\section{References}

BaILey, H. (1958) Emergency Surgery. Wright, Bristol.
Desmond, A.M. \& HutTER, F. (1948) Strangulated obturator hernia. Brit. J. Surg. 35, 318.

HaRPER, J.R. \& Holt, J.H. (1956) Obturator hernia. Amer. J. Surg. 92, 562.

KWONG, K.H. \& ONG, G.B. (1966) Obturator hernia. Brit. J. Surg. 53, 23.

WAKELEY, C.P.G. (1939) Obturator hernia. Brit. J. Surg. 26, 515.

Watson, L. F. (1948) Hernia, 3rd edn., p. 457. Mosby, London.

.

